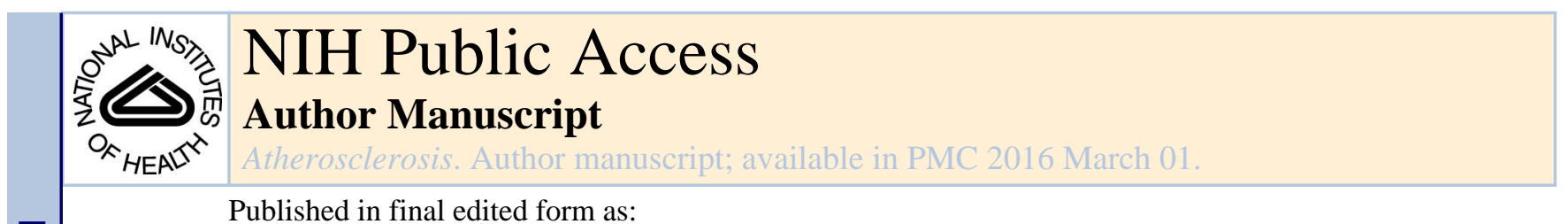

Published in final edited form as:

Atherosclerosis. 2015 March ; 239(1): 101-108. doi:10.1016/j.atherosclerosis.2014.12.044.

\title{
The Association of Resistin with Cardiovascular Disease in The Multi-Ethnic Study of Atherosclerosis
}

\author{
Evan D. Muse, MD, PhD ${ }^{1,2}$, David I. Feldman, BS ${ }^{1}$, Michael J. Blaha, MD, MPH ${ }^{1}$, Zeina \\ Dardari, MS ${ }^{1}$, Roger S. Blumenthal, MD ${ }^{1}$, Matthew J. Budoff, MD ${ }^{3}$, Khurram Nasir, MD, \\ $\mathrm{MPH}^{1,4}$, Michael H. Criqui, MD, MPH${ }^{5}$, Mary Cushman, MD, $\mathrm{MSc}^{6}$, Robyn L. McClelland, \\ $\mathrm{PhD}^{7}$, and Matthew A. Allison, MD, $\mathbf{M P H}^{5}$ \\ ${ }^{1}$ Johns Hopkins Ciccarone Center for the Prevention of Heart Disease, Baltimore, MD \\ ${ }^{2}$ Scripps Translational Science Institute, The Scripps Research Institute, La Jolla, CA \\ ${ }^{3}$ Division of Cardiology, Harbor-UCLA Medical Center, Los Angeles, CA \\ ${ }^{4}$ Center for Prevention and Wellness Research, Baptist Health Medical Group, Miami, FL \\ ${ }^{5}$ Department of Family and Preventive Medicine, University of California San Diego, San Diego, \\ $\mathrm{CA}$ \\ ${ }^{6}$ Department of Medicine, University of Vermont, Burlington, VT \\ ${ }^{7}$ Department of Biostatistics, University of Washington, Seattle, WA
}

\section{Abstract}

Objective-To describe the relationship between circulating resistin levels and cardiovascular diseases (CVD) and all-cause death in a multi-ethnic cohort.

Methods and Results-We studied 1,913 participants from the Multi-Ethnic Study of Atherosclerosis with measurements of plasma resistin levels. Absolute proportions experiencing new-onset atrial fibrillation (AF), atherosclerotic CVD (myocardial infarction, angina, resuscitated cardiac arrest, stroke), heart failure (HF), and all-cause death were calculated for each quartile of resistin. We used adjusted Cox proportional regression modeling resistin as a continuous variable per standard deviation of log-transformed resistin and secondarily as a categorical variable using resistin quartiles. Results were stratified by sex and race/ethnicity. The mean age of the population was $64.5 \pm 10$ years with half being female and a median resistin concentration of $15.1 \mathrm{ng} / \mathrm{mL}$ (11.9-19.1). Mean follow-up time was $7.2 \pm 1.8$ years. There was a graded increase in the occurrence of all outcomes across increasing quartiles of resistin. Modeled as a continuous variable, after adjustment for anthropomorphic measures, traditional risk factors, markers of

(C) 2014 Elsevier Ltd. All rights reserved.

Corresponding Author: Evan D. Muse, Scripps Translational Science Institute, 3344 N. Torrey Pines Court, Suite 300, La Jolla, CA 92037, (858) 554-5751, emuse@ scripps.edu.

Publisher's Disclaimer: This is a PDF file of an unedited manuscript that has been accepted for publication. As a service to our customers we are providing this early version of the manuscript. The manuscript will undergo copyediting, typesetting, and review of the resulting proof before it is published in its final citable form. Please note that during the production process errors may be discovered which could affect the content, and all legal disclaimers that apply to the journal pertain.

Disclosures: None of the authors report conflicts of interest pertaining to this work. 
inflammation, and other adipokines, significant associations were noted for HF (HR 1.4, CI 1.02.0), hard and all CVD (HR 1.3, 1.1-1.7 and 1.3, 1.1-1.6, respectively), and CHD (HR 1.31, 1.01.6), but not for AF or death. Significant interaction terms were noted between resistin and race, with Hispanic race/ethnicity showing the strongest relationship between resistin and outcomes.

Conclusions-In an ethnically diverse population without known CVD at baseline, there was a strong, independent association between higher resistin levels and incident CVD, CHD and HF.

\section{Keywords}

atherosclerotic heart disease; risk stratification; resistin; adipokines

\section{INTRODUCTION}

Despite advances in our understanding of the major modifiable risk factors for cardiovascular diseases (CVD), these diseases remain the leading causes of morbidity and mortality worldwide ${ }^{1,2}$. The increase in the prevalence of obesity, with its subsequent effects on cardiometabolic risk factors (e.g. hypertension, dyslipidemia, insulin resistance) has emerged as a critical obstacle to effective CVD prevention ${ }^{3}$. Obesity and metabolic disease also persist as a key contributing factor to other diseases such as atrial fibrillation (AF). As part of the ongoing efforts for preventing CVD a concerted effort has been made to identify novel biomarkers of obesity-related risk to improve CVD risk prediction and help better understand the pathophysiology of this disease process.

One such candidate marker is the circulating $12.5-\mathrm{kDa}$ cysteine-rich, polypeptide resistin. Resistin is a member of the FIZZ (found in inflammatory zones) family of proteins and belongs to a group of adipose tissue-related hormones (termed adipokines) that includes leptin, adiponectin, adipsin and visfatin ${ }^{4-6}$, among others. In rodents, higher plasma resistin levels from models of diet-induced obesity are associated with concomitant increases in insulin resistance ${ }^{7-9}$. Human resistin, however, shares only 55\% amino acid homology with that of rodents and is expressed mainly by peripheral blood mononuclear cells, macrophages, and bone marrow. This may explain why reports of the relationship of plasma resistin levels to obesity in humans have been conflicting ${ }^{4}$. However, the relationship of resistin to classic mediators of inflammation, such as interleukin-6 (IL-6) and tumor necrosis factor alpha (TNF-a), appears more consistent ${ }^{10-15}$. In fact, resistin has been shown to have pleiotropic functions in metabolism and physiology with roles in inflammation ${ }^{16}$, endothelial dysfunction ${ }^{17,18}$, cardiomyocyte function ${ }^{19}$, and cholesterol metabolism ${ }^{20}$.

Prior studies of the association between plasma resistin levels and CVD have been conflicting ${ }^{21-25}$. This may be partially explained by limitations posed by small sample sizes and limited ethnic diversity. Therefore, we aimed to further describe the relationship between circulating resistin levels and CVD outcomes in the racially diverse, sex-balanced participants of the Multi-Ethnic Study of Atherosclerosis (MESA). 


\section{MATERIALS AND METHODS}

\section{Study Design}

The Multi-Ethnic Study of Atherosclerosis (MESA) is a longitudinal cohort study aimed at identifying the determinants of cardiovascular disease among four ethnic groups in the United States. Details of the MESA study design have been previously published ${ }^{26}$. In brief, between July 2000 and August 2002, MESA enrolled a total of 6814 men and women aged 45-84 years, who were free of clinically apparent cardiovascular disease (CVD) from six communities geographically dispersed throughout the United States. Participants were recruited at each site from lists of residents, dwellings, and telephone-company customers with emphasis on ethnic diversity. Individuals with a history of physician-diagnosed heart attack, angina, heart failure, stroke or transient ischemic attack (TIA), or having undergone an invasive procedure for CVD were excluded from participation.

\section{Study Population}

At clinic visits 2 and 3 (2002-2005), a random subsample of 1,970 participants

(approximately one half at each visit) enrolled in an ancillary study that obtained computed tomography scans of the abdomen to address hypotheses including the associations between body composition, inflammation, and CVD, as well as biomarkers from fasting venous blood samples. The present study population consists of 1,913 participants with measures of plasma resistin obtained from these samples and long-term follow-up for CVD events.

\section{Clinical and Laboratory Measurements}

At all clinic visits, standardized questionnaires were administered to obtain sociodemographic, ethnicity, and health history information. Body mass index (BMI) was calculated by dividing the weight kilograms by the square of the height in meters. Waist circumferences were measured using a standard flexible tape measure. Hypertension was defined according to Joint National Committee 6 (JNC-6) criteria. Diabetes was defined according to American Diabetes Association (ADA) guidelines as a fasting plasma glucose level $>7 \mathrm{mmol} / \mathrm{L}$ or a history of medical treatment for diabetes.

At each clinic visit and after a 12-hour fast, venous blood was collected and shipped to the MESA central laboratory for measurement of total and HDL cholesterol, triglycerides, and glucose levels. Fasting blood was also assayed for high sensitivity C-reactive protein (hsCRP) and insulin concentration. Homeostatic Model Assessment of Insulin Resistance (HOMA-IR) was calculated as fasting insulin $[\mu \mathrm{U} / \mathrm{mL}] \times$ fasting glucose $[\mathrm{mg} / \mathrm{dL}] / 405$. The presence of microalbuminuria, defined as 30-300 microgram albumin per milligram creatinine, was determined by spot urinalysis.

Adipokines including adiponectin, leptin, and resistin were measured using stored EDTA plasma samples from MESA visits 2 and 3. Each adipokine was measured using Bio-Rad Luminex flow cytometry (Millepore, Billerica, MA) at the Laboratory for Clinical Biochemistry Research (University of Vermont, Burlington, VT). Average analytic coefficients of variation across several control samples for these analytes ranged from $6.0 \%$ to $13.0 \%$. 


\section{Follow-up and Event Adjudication}

Starting at baseline, new cases of atrial fibrillation (AF), heart failure (HF), coronary heart disease (CHD), cardiovascular disease (CVD), and all-cause mortality were recorded over a mean of $7.2 \pm 1.8$ years. At intervals of 9 to 12 months, an interviewer contacted each participant or a family member regarding interim hospital admissions, outpatient diagnoses, and deaths. Two physician adjudicators independently classified events, and in the event of disagreement, the full committee adjudicated. AF was determined by ICD-9 code from hospitalizations, death certificates or by participant self-report.

HF events required heart failure symptoms, a physician diagnosis of HF, and initiation of medical therapy for HF. CHD events consisted of myocardial infarction, death from coronary heart disease, definite angina, probable angina followed by coronary revascularization, or resuscitated cardiac arrest. CVD events consisted of CHD events plus stroke (not transient ischemic attacks), stroke death, other atherosclerotic death, and other CVD death. "Hard" outcomes excluded angina and revascularization. A detailed description of the MESA follow-up methods is available at www.mesa-nhlbi.org. Baseline for this substudy was considered at the time of abdominal CT that occurred on visit 2 or visit 3 .

\section{Statistical Analysis}

Participants were divided into quartiles based on plasma resistin levels. Characteristics are presented for each quartile and differences across quartiles were calculated using chi-square tests (for categorical variables), and analysis-of-variance tests (for continuous, normally distributed variables) or Kruskal-Wallis equality of populations rank tests (for continuous skewed variables). Given the right-skewed distribution of resistin data, box plots were generated displaying the median, interquartile range, and $95 \%$ range of resistin values by both sex and race/ethnicity.

Crude proportions of participants experiencing each outcome were calculated across resistin quartiles. We then analyzed time-to-event data using Cox proportional hazards models. The continuous association between plasma resistin and each outcome was evaluated using restricted cubic splines, centered at the median resistin value. Resistin was then modeled as a continuous variable per standard deviation of log-transformed resistin. In additional analyses, resistin was also modeled as a categorical variable using resistin quartiles. The proportionality assumption was graphically confirmed using both the log-log plot of survival and by comparison of the Kaplan-Meier and predicted survival plots. Models were adjusted for demographics, measures of adipose-related risk, traditional risk factors, and other adipokines as follows:

Model 1 - age, sex, and race

Model 2 - Model $1+$ hypertension (and antihypertensive therapy), diabetes, smoking, total cholesterol, HDL cholesterol, lipid lowering therapy

Model 3 - Model 2 + BMI, HOMA-IR, log-transformed hsCRP

Model 4 - Model 3 + adiponectin, leptin 
Additional exploratory analyses were conducted within race/ethnicity-specific subgroups, including formal interaction testing. In these analyses, which were limited by small sample size, interactions were tested with covariates from only Models $1 \& 2$ to limit model overfitting.

\section{RESULTS}

The mean [SD] and median [IQR] resistin levels were $16.3[6.8 \mathrm{ng} / \mathrm{mL}]$ and 15.1 [11.9-19.1 $\mathrm{ng} / \mathrm{mL}]$, respectively. Characteristics of the study participants across quartiles of resistin are displayed in Table 1. Across increasing quartiles of resistin, there was a significant difference in age, race, and socio-economic status ( $\mathrm{P}<0.01)$, but no significant difference in $\operatorname{sex}(\mathrm{P}=0.12)$. Individual risk factors such as $\mathrm{BMI}$, waist circumference, and systolic blood pressure showed significant increases across increasing resistin quartiles $(\mathrm{P}<0.01)$ whereas HDL-cholesterol decreased significantly $(\mathrm{P}<0.01)$. Similarly, the Framingham risk score increased across plasma resistin quartiles ( $\mathrm{Q} 1$ vs. Q4: $7.7 \pm 6.5$ vs. $9.8 \pm 7.6 \%, \mathrm{P}<0.01)$, as did the percentage of individuals on antihypertensive medication $(\mathrm{P}<0.01)$, while the proportion on lipid-lowering therapies was not different $(\mathrm{P}=0.42)$. In addition, across increasing quartiles of resistin, there was a significant increase in both HOMA-IR and hsCRP $(\mathrm{P}<0.01)$, with statistically significant correlation coefficients seen for both measures in men and women.

There were 962 (50.3\%) female and 951 (49.7\%) male participants. Median resistin levels were not significantly different between males and females [15.2 vs. $14.8 \mathrm{ng} / \mathrm{mL}, \mathrm{p}=0.24)$ ] (Supplemental Figure 1A). The median resistin levels were significantly different $(\mathrm{p}<0.01)$ by race/ethnicity: Caucasian $(15.2 \mathrm{ng} / \mathrm{mL})$, Chinese (14.0 ng/mL), African American (15.4 $\mathrm{ng} / \mathrm{mL}$ ), and Hispanic subjects (15.1 ng/mL) (Supplemental Figure 1B). Among Caucasians, males $(16.0 \pm 5.0 \mathrm{ng} / \mathrm{mL})$ and females $(16.1 \pm 5.5 \mathrm{ng} / \mathrm{mL})$ showed similar median levels of plasma resistin. In Chinese, who reported the lowest overall mean resistin concentrations, males $(15.9 \pm 7.7 \mathrm{ng} / \mathrm{mL})$ had higher resistin concentrations than females $(14.5 \pm 7.2 \mathrm{ng} /$ $\mathrm{mL})$. Conversely, African Americans had the highest levels of plasma resistin, with men $(17.6 \pm 9.1 \mathrm{ng} / \mathrm{mL})$ and women $(17.4 \pm 8.7 \mathrm{ng} / \mathrm{mL})$ having similar levels. A large gap between sexes also existed in Hispanics, with men $(14.9 \pm 5.1 \mathrm{ng} / \mathrm{mL})$ being significantly lower than women $(17.3 \pm 7.7 \mathrm{ng} / \mathrm{mL})$.

Figure 1 displays the percentage of participants experiencing the specified cardiovascular events/outcomes across increasing plasma resistin quartiles. In general, there was a graded increase in event rates across the first to the fourth quartile. For the $4^{\text {th }}$ quartile of resistin, $3.9 \%$ of participants developed AF, accounting for 18 of the 58 total AF events; $5.3 \%$ ( 25 of the 52) of participants developed HF; $10.8 \%$ (51 of the 148) of participants developed allcause death; 8.5\% (40 of the 110) of participants developed hard CVD events; $11.2 \%$ (53 of 153) of participants developed total CVD and $8.2 \%$ (39 of 107) of participants developed CHD. Composite event numbers for each outcome (Table 2) and total event numbers by race and sex were also determined (Supplemental Table 1).

In an unadjusted analysis of resistin as a continuous variable, a one standard deviation increase in log-transformed resistin was associated with a statistically significant $(\mathrm{p}<0.05)$ 
increase in risk (HR, 95\% CI) of HF (1.9, 1.5-2.5), CVD (hard) (1.5, 1.3-1.8), CVD (all) $(1.5,1.2-1.7)$, CHD $(1.5,1.2-1.8)$ and death $(1.3,1.1-1.5)$ (Table 2$)$. The risk of death with increasing resistin levels was no longer significant after adjusting for age, sex and race (Model 1). However, after step-wise multivariable adjustment for traditional cardiovascular risk factors (from Model 1 through Model 4) the risk of HF (1.4, 1.0-2.0), CVD (hard) (1.3, 1.1-1.7), CVD (all) (1.3, 1.1-1.6) and CHD (1.3, 1.0-1.6) remained significantly elevated persisting even in the fully adjusted model (Model 4).

To facilitate comparison with prior literature, secondary multivariable hazard ratios (HR) for the $4^{\text {th }}$ quartile of resistin vs. the $1^{\text {st }}$ quartile were assessed in five hierarchical regression models (Unadjusted, Model 1,2,3, and 4) for AF, HF, death, CVD (hard events), CVD (all events), and CHD (Supplemental Table 2). The strongest association was observed for $4^{\text {th }}$ quartile vs $1^{\text {st }}$ quartile resistin levels and CHD with an unadjusted HR of 3.5 (1.8-6.6), and retaining significance $(\mathrm{P}<0.05)$ after full multivariable adjustment $(2.2,1.1-4.5)$. Although there was a general trend for the highest resistin levels to be associated with a greater risk of developing AF (Supplemental Table 2), this association failed to achieve statistical significance in an unadjusted model $(1.9,0.9-4.0)$. Similarly, for HF, mortality, CVD (hard) and CVD (all) events, the HR for the unadjusted models was significantly elevated but did not maintain significance after adjustment for traditional risk factors.

Given the multi-ethnic heritage of MESA, we further assessed the race-specific effects in the association of resistin with cardiovascular outcomes. In a race-specific analysis (Table 3), statistical power for most outcomes was lost due to a small number of events in patient subsets (Supplemental Table 1). To limit over-fitting in our models, we restricted our multivariable adjustment to models 1 and 2 for each subset. Even with restricted adjustment, the hazard ratios for Chinese and Black participants failed to reach statistical significance in either model while only HF (HR 1.8, 1.0-3.2) remained significant in Caucasians. However, there remained a significant increased risk for HF (HR 1.9, 1.1-3.4), CVD (hard) (HR 2.1, 1.4-3.1), CVD (all) (HR 1.8, 1.2-2.5) and CHD (HR 1.5, 1.0-2.4) in Hispanics after adjustments in model 2 . In an overall race interaction analysis, the interaction term was significant for CVD (hard) after adjustments for variables in model $1(\mathrm{p}=0.02)$ and in model 2 ( $\mathrm{p}=0.02)$. This appears to be driven by more significant findings in Hispanics. As such, the interaction term for Hispanic vs. non-Hispanic X SD log [resistin] was significant for both CVD (hard) and CVD (all) after adjustments for variables in model 1 ( $\mathrm{p}=0.01$ in both) and in model 2 ( $\mathrm{p}=0.03$ in both).

Restricted cubic spline analysis was conducted for each outcome across continuous plasma concentrations of resistin after adjustment for age, sex, hypertension (and antihypertensive therapy), diabetes, smoking, total cholesterol, HDL cholesterol, lipid lowering therapy (according to Model 2; Materials and Methods) to assess the shape of the associations and to assess for potential threshold effects (Figure 2). A clear trend for increased events for all outcomes assessed was observed with increasing concentrations of resistin, without clear evidence of a threshold effect. 


\section{DISCUSSION}

In this racially diverse cohort of patients from the Multi-Ethnic Study of Atherosclerosis (MESA), there was a robust association of increasing resistin with elevated incidence of HF, all and hard CVD, and CHD events independent of traditional cardiovascular risk factors, obesity, markers of inflammation/insulin resistance, and several adipokines. While the association of resistin levels with AF and death followed a similar trend, the associations were not significant after multi-variable adjustment. Since these findings illustrate the complex interplay between adiposity, inflammation, and traditional risk factors in mediating adverse cardiovascular outcomes, the pathophysiologic importance of resistin should be considered.

Although resistin has been measured in several prior single ethnicity cohorts, this is the first study to directly compare the association of resistin levels with outcomes in a multi-ethnic population. Given the rigorous collection of prospective events data in MESA, we were able to assess the association of resistin with six specific cardiovascular events in our analysis. Additionally, given the expansive phenotyping, MESA also allows for adjustment for numerous potentially confounding markers, for example HOMA-IR, hs-CRP, and other adipokines.

MESA also highlights the importance of comprehensive multivariable adjustment in the study of the prognostic significance of resistin. We observed that individuals belonging to the highest quartile of resistin had higher levels of the traditional CVD risk factors (hypertension, diabetes mellitus, microalbuminuria, lower socioeconomic status, smoking, and greater waist circumference). A lingering question in the literature has been, are the elevations of resistin in these patients resulting from a state of chronic low-grade inflammatory disease (passive biomarker) or does resistin play a causal role in the disease process (active agent)? While this certainly deserves further study, we have shown that the risk of developing CHD events remained at least double in this cohort for patients in the highest quartile of resistin even after a fully adjusted multivariable analysis. There was no significant change in these adjusted models after sensitivity analysis were done that replaced hypertension with systolic or diastolic blood pressures.

Our study adds to the findings of a 2012 Reinstra et al. in the Framingham Offspring Study (FOS), which examined a community-based population for the association of resistin with the incidence of $\mathrm{AF}^{27}$. They concluded that higher mean resistin concentrations were associated with incident AF, but the relation was attenuated after adjusting for hs-CRP. In a more diverse population and with a shorter follow up period (5.5 vs. 7.6 years), we observed fewer total AF events compared to the FOS (58 vs. 206) ${ }^{27}$. We did not find an independent association with concentrations of resistin and incident AF in any model, including an unadjusted model, but may have been underpowered given the low event rate for this outcome. This remained unchanged after additional analyses were conducted that adjusted for health insurance status.

In another study of the Framingham Offspring examining the ability of resistin to predict cardiovascular events, Frankel et al. described the association of increased plasma resistin 
levels and subsequent development of $\mathrm{HF}^{21}$. This study reported a persistent association between resistin and incident HF in the upper $3^{\text {rd }}$ tertile of resistin $(5.2,1.08-25.13)$. In addition to the overwhelming majority of Caucasians in their population (99\%), their data did not allow for adjustment for markers (i.e. insulin therapy, waist circumference, lipidlowering therapy, leptin, adiponectin) that are suggestive mediators in the association between resistin and HF. In comparison, when looking at the ethnically diverse MESA study, we found that the association between resistin and incident HF was no longer significant after adjusting for CVD risk factors and several adipokines.

Our study provides further support of the relationship between elevated resistin and HF events in this multi-ethnic population, as has been described in other populations ${ }^{21,28,29}$. However, the strength of this relationship was attenuated after adjusting for traditional cardiovascular risk factors, a concept also appreciated by other groups ${ }^{30}$. This may be secondary to confounding factors or that the outcome is mediated by covariates in our model, as the event rate was sufficient (52 total HF events) in the current study. In support of our findings, Bobbert and colleagues have reported the increase of adverse clinical events for patients with cardiomyopathy, which is associated with higher levels of circulating resistin levels ${ }^{31}$. On this same spectrum, heart failure patients destined for left ventricular assist device (LVAD) therapy show elevated resistin levels compared to the control groups until LVAD implantation, after which implantation resulted in a downward trend in plasma resistin ${ }^{32}$.

The association of adipokines to left ventricular hypertrophy in this same cohort of patients in the MESA study was previously assessed ${ }^{33}$. There, resistin was found to be the most powerful marker of decreased ejection fraction and a higher odds of left ventricular (LV) hypertrophy as measured by cardiac magnetic resonance imaging. Others have reported an association between increased plasma resistin and reduced LV fractional shortening as measured by transthoracic echocardiography ${ }^{34}$. These findings are in support of preclinical evidence that illustrates the effect of resistin on caridomyocyte growth and impaired contractile function, and points to potential mechanisms for the development of heart failure ${ }^{19}$.

In an attempt to describe possible mechanisms for resistin and its role in mediating specific cardiovascular events, a detailed analysis of its origin and its association with different markers of inflammation and obesity is crucial. Resistin, which originates from monocytes/ macrophages in humans and not from adipose tissue as in rodents, has fallen short of its original expectation of being the causal link between obesity and insulin resistance. It is now appreciated that obesity does in fact represent a state of chronic, low-grade inflammation in which circulating resistin is found to be oftentimes elevated. Moreover, increased circulating resistin levels are also associated with numerous inflammatory diseases such as rheumatoid arthritis, ulcerative colitis, and certain malignancies ${ }^{35}$.

Although inflammation is thought to contribute to incident AF, and a small study from Turkey reporting that increased resistin was related to paroxysmal and persistent AF, although not permanent $\mathrm{AF}^{36}$, we did not see an independent association of resistin with $\mathrm{AF}$ in this cohort during the follow-up period. Our ability to fully investigate AF as an outcome, 
however, was limited in that AF is not a fully adjudicated outcome in MESA but a selfreported or ICD-9 based event from inpatient records.

While it is documented that inflammation can serve as a mechanism for linking resistin to adverse cardiovascular outcomes, it is not considered the only pathway for the involvement of resistin in $\mathrm{CVD}^{27}$. Prior studies have shown resistin to be linked to both endothelial and cardiomyocyte dysfunction, as well as alterations in cholesterol metabolism. Accordingly, the association of resistin with subclinical atherosclerosis has been illustrated by its relationship to elevated coronary artery calcium levels ${ }^{11}$ and increased carotid intima-media thickness ${ }^{37}$. Our study extends beyond subclinical atherosclerosis and focuses in on CVD events. In addition to our findings associating increased resistin with ischemic stroke, Choi and colleagues showed that increased vascular inflammation, as measured by whole body FDG-PET imaging, is strongly associated with increased circulating resistin levels. This lends further support to the intricate relationship between resistin and inflammation in the pathogenesis of $\mathrm{CVD}^{38}$.

While the association between resistin and cardiovascular events was the strongest in Hispanics, given the small number of events, we were unable to draw definitive conclusions about our ethnicity-specific subgroup analyses. As an epidemiologic study, we are limited by our inability to fully discern the mechanisms by which resistin contributes to the development of cardiovascular events. Lastly, additional confounding factors may exist that remain unidentified.

In conclusion, our study demonstrates a robust association between increased levels of plasma resistin and HF, CVD (all and hard) and CHD outcomes in a large, multi-ethnic population. Our data will be of great interest to those studying the importance of resistin in human disease, especially in a Hispanic population. However, additional biomarker validation studies must be done to substantiate a clinical value to measuring plasma resistin in patients.

\section{Supplementary Material}

Refer to Web version on PubMed Central for supplementary material.

\section{Acknowledgments}

The authors thank the other investigators, the staff, and the participants of the MESA study for their valuable contributions. A full list of participating MESA investigators and institutions can be found at http://www.mesanhlbi.org. The information contained herein was derived in part from data provided by the Bureau of Vital Statistics, New York City Department of Health and Mental Hygiene.

Sources of Funding: This research was supported by contracts N01-HC-95159, N01-HC-95160, N01-HC-95161, N01-HC-95162, N01-HC-95163, N01-HC-95164, N01-HC-95165, N01-HC-95166, N01-HC-95167, N01HC-95168 and N01-HC-95169 from the National Heart, Lung, and Blood Institute and by grants UL1-TR-000040 and UL1-TR-001079 from NCRR. EDM is supported by 8KL2-TR-000110 from the National Institutes of Health.

\section{References}

1. Murray CJL, Lopez AD. Measuring the global burden of disease. N Engl J Med. 2013; 369(5):44857.10.1056/NEJMra1201534 [PubMed: 23902484] 
2. Go AS, Mozaffarian D, Roger VL, et al. Heart Disease and Stroke Statistics-2014 Update: A Report From the American Heart Association. Circulation. 2013; 129(3):e28-e292.10.1161/01.cir. 0000441139.02102.80 [PubMed: 24352519]

3. Lavie CJ, Milani RV, Ventura HO. Obesity and cardiovascular disease: risk factor, paradox, and impact of weight loss. J Am Coll Cardiol. 2009; 53(21):1925-32.10.1016/j.jacc.2008.12.068 [PubMed: 19460605]

4. Lee SE, Kim H-S. Human resistin in cardiovascular disease. J Smooth Muscle Res. 2012; 48(1):2735. [PubMed: 22504487]

5. Schwartz D, Lazar M. Human resistin: found in translation from mouse to man. Trends Endocrinol Metab. 2011; 22(7):259-265.10.1016/j.tem.2011.03.005.Human [PubMed: 21497511]

6. Gualillo O, González-Juanatey JR, Lago F. The emerging role of adipokines as mediators of cardiovascular function: physiologic and clinical perspectives. Trends Cardiovasc Med. 2007; 17(8):275-83.10.1016/j.tcm.2007.09.005 [PubMed: 18021938]

7. Steppan CM, Bailey ST, Bhat S, et al. The hormone resistin links obesity to diabetes. Nature. 2001; 409:307-312.10.1038/35053000 [PubMed: 11201732]

8. Steppan CM, Lazar Ma. The current biology of resistin. J Intern Med. 2004; 255(4):439_ 47.10.1111/j.1365-2796.2004.01306.x [PubMed: 15049878]

9. Muse ED, Obici S, Bhanot S, et al. Role of resistin in diet-induced hepatic insulin resistance. J Clin Invest. 2004; 114(2):232-9.10.1172/JCI21270 [PubMed: 15254590]

10. Kaser S, Kaser A, Sandhofer A, Ebenbichler CF, Tilg H, Patsch JR. Resistin messenger-RNA expression is increased by proinflammatory cytokines in vitro. Biochem Biophys Res Commun. 2003; 309:286-290.10.1016/j.bbrc.2003.07.003 [PubMed: 12951047]

11. Reilly MP, Lehrke M, Wolfe ML, Rohatgi A, Lazar Ma, Rader DJ. Resistin is an inflammatory marker of atherosclerosis in humans. Circulation. 2005; 111(7):932-9.10.1161/01.CIR. 0000155620.10387.43 [PubMed: 15710760]

12. Shetty GK, Economides PA, Horton ES, Mantzoros CS, Veves A. Circulating adiponectin and resistin levels in relation to metabolic factors, inflammatory markers, and vascular reactivity in diabetic patients and subjects at risk for diabetes. 2004:2450-2457.10.2337/diacare.27.10.2450

13. Bokarewa M, Nagaev I, Dahlberg L, Smith U, Tarkowski A. Resistin, an adipokine with potent proinflammatory properties. J Immunol. 2005; 174:5789-5795.10.1042/CS20050078 [PubMed: 15843582]

14. Lehrke M, Reilly MP, Millington SC, Iqbal N, Rader DJ, Lazar MA. An inflammatory cascade leading to hyperresistinemia in humans. PLoS Med. 2004; 1(2):e45.10.1371/journal.pmed. 0010045 [PubMed: 15578112]

15. Qasim AN, Metkus TS, Tadesse M, et al. Resistin gene variation is associated with systemic inflammation but not plasma adipokine levels, metabolic syndrome or coronary atherosclerosis in nondiabetic Caucasians. Clin Endocrinol (Oxf). 2009; 70(5):698-705.10.1111/j. 1365-2265.2008.03375.x [PubMed: 18710472]

16. Aquilante CL, Kosmiski La, Knutsen SD, Zineh I. Relationship between plasma resistin concentrations, inflammatory chemokines, and components of the metabolic syndrome in adults. Metabolism. 2008; 57(4):494-501.10.1016/j.metabol.2007.11.010 [PubMed: 18328350]

17. Ntaios G, Gatselis NK, Makaritsis K, Dalekos GN. Adipokines as mediators of endothelial function and atherosclerosis. Atherosclerosis. 2013; 227(2):216-21.10.1016/j.atherosclerosis. 2012.12.029 [PubMed: 23332774]

18. Burnett MS, Lee CW, Kinnaird TD, et al. The potential role of resistin in atherogenesis. Atherosclerosis. 2005; 182(2):241-8.10.1016/j.atherosclerosis.2005.02.014 [PubMed: 16159596]

19. Kim M, Oh JK, Sakata S, et al. Role of resistin in cardiac contractility and hypertrophy. J Mol Cell Cardiol. 2008; 45(2):270-80.10.1016/j.yjmcc.2008.05.006 [PubMed: 18597775]

20. Melone M, Wilsie L, Palyha O, Strack A, Rashid S. Discovery of a new role of human resistin in hepatocyte low-density lipoprotein receptor suppression mediated in part by proprotein convertase subtilisin/kexin type 9. J Am Coll Cardi ol. 2012; 59(19):1697-705.10.1016/j.jacc.2011.11.064

21. Frankel DS, Vasan RS, D'Agostino RB, et al. Resistin, adiponectin, and risk of heart failure the Framingham offspring study. J Am Coll Cardiol. 2009; 53(9):754-62.10.1016/j.jacc.2008.07.073 [PubMed: 19245965] 
22. Lim S, Koo BK, Cho SW, et al. Association of adiponectin and resistin with cardiovascular events in Korean patients with type 2 diabetes: the Korean atherosclerosis study (KAS): a 42-month prospective study. Atherosclerosis. 2008; 196(1):398-404.10.1016/j.atherosclerosis.2006.11.017 [PubMed: 17178123]

23. Luc G, Empana J-P, Morange P, et al. Adipocytokines and the risk of coronary heart disease in healthy middle aged men: the PRIME Study. Int J Obes (Lond). 2010; 34(1):118-26.10.1038/ijo. 2009.204 [PubMed: 19823188]

24. Menzaghi C, Bacci S, Salvemini L, et al. Serum resistin, cardiovascular disease and all-cause mortality in patients with type 2 diabetes. PLoS One. 2013; 8(6):e64729.10.1371/journal.pone. 0064729 [PubMed: 23755138]

25. Yin W-H, Wei J, Huang W-P, Chen J-W, Young MS, Lin S-J. Prognostic value of circulating adipokine levels and expressions of adipokines in the myocardium of patients with chronic heart failure. Circ J. 2012; 76(9):2139-47.10.1253/circj.CJ-11-1549 [PubMed: 22785032]

26. Bild DE, Bluemke DA, Burke GL, et al. Multi-ethnic study of atherosclerosis: objectives and design. Am J Epidemiol. 2002; 156(9):871-81.10.1093/aje/kwf113 [PubMed: 12397006]

27. Rienstra M, Sun JX, Lubitz Sa, et al. Plasma resistin, adiponectin, and risk of incident atrial fibrillation: the Framingham Offspring Study. Am Heart J. 2012; 163(1):119-124.e1.10.1016/j.ahj. 2011.09.029 [PubMed: 22172445]

28. Butler J, Kalogeropoulos A, Georgiopoulou V, et al. Serum resistin concentrations and risk of new onset heart failure in older persons: the health, aging, and body composition (Health ABC) study. Arterioscler Thromb Vasc Biol. 2009; 29(7):1144-9.10.1161/ATVBAHA.109.186783 [PubMed: 19372460]

29. Schulze PC, Biolo A, Gopal D, et al. Dynamics in insulin resistance and plasma levels of adipokines in patients with acute decompensated and chronic stable heart failure. J Card Fail. 2011; 17(12):1004-11.10.1016/j.cardfail.2011.08.010 [PubMed: 22123363]

30. Zhang MH, Na B, Schiller NB, Whooley MA. Association of resistin with heart failure and mortality in patients with stable coronary heart disease: data from the heart and soul study. $\mathrm{J}$ Card Fail. 2011; 17(1):24-30.10.1016/j.cardfail.2010.08.007 [PubMed: 21187261]

31. Bobbert P, Jenke A, Bobbert T, et al. High leptin and resistin expression in chronic heart failure: adverse outcome in patients with dilated and inflammatory cardiomyopathy. Eur J Heart Fail. 2012; 14(11):1265-75.10.1093/eurjhf/hfs111 [PubMed: 22764185]

32. Khan RS, Kato TS, Chokshi A, et al. Adipose tissue inflammation and adiponectin resistance in patients with advanced heart failure: correction after ventricular assist device implantation. Circ Heart Fail. 2012; 5(3):340-8.10.1161/CIRCHEARTFAILURE.111.964031 [PubMed: 22379072]

33. Allison MA, Bluemke DA, McClelland R, et al. Relation of leptin to left ventricular hypertrophy (from the Multi-Ethnic Study of Atherosclerosis). Am J Cardiol. 2013; 112(5):726-30.10.1016/ j.amjcard.2013.04.053 [PubMed: 23711806]

34. McManus DD, Lyass A, Ingelsson E, et al. Relations of circulating resistin and adiponectin and cardiac structure and function: the Framingham Offspring Study. Obesity (Silver Spring). 2012; 20(9):1882-6.10.1038/oby.2011.32 [PubMed: 21350435]

35. Filková M, Haluzík M, Gay S, Senolt L. The role of resistin as a regulator of inflammation: Implications for various human pathologies. Clin Immunol. 2009; 133(2):157-70.10.1016/j.clim. 2009.07.013 [PubMed: 19740705]

36. Özcan KS, Güngör B, Altay S, et al. Increased level of resistin predicts development of atrial fibrillation. J Cardiol. 2014; 63(4):308-12.10.1016/j.jjcc.2013.10.008 [PubMed: 24268420]

37. Rubio-Guerra AF, Cabrera-Miranda LJ, Vargas-Robles H, Maceda-Serrano A, Lozano-Nuevo JJ, Escalante-Acosta $\mathrm{Ba}$. Correlation between levels of circulating adipokines and adiponectin/resistin index with carotid intima-media thickness in hypertensive type 2 diabetic patients. Cardiology. 2013; 125(3):150-3.10.1159/000348651 [PubMed: 23736118]

38. Choi HY, Kim S, Yang SJ, et al. Association of adiponectin, resistin, and vascular inflammation: analysis with 18F-fluorodeoxyglucose positron emission tomography. Arterioscler Thromb Vasc Biol. 2011; 31(4):944-9.10.1161/ATVBAHA.110.220673 [PubMed: 21212400] 


\section{SIGNIFICANCE}

Given the increased prevalence of obesity and subsequent increased risk of cardiovascular disease efforts have been made to identify novel biomarkers of obesityrelated risk for better cardiovascular disease risk prediction. In this multi-ethnic cohort of asymptomatic patients we assessed the relationship between circulating resistin levels and cardiovascular outcomes over a 7-year period. We observed a strong, independent association between increasing resistin levels and cardiovascular disease, coronary heart disease and heart failure. 


\section{HIGHLIGHTS}

- In an asymptomatic, multi-ethnic population we examined the association of circulating resistin levels and cardiovascular disease.

- Events included new onset atrial fibrillation, myocardial infarction, angina, resuscitated cardiac arrest, stroke, heart failure and all-cause death.

- Significant associations of increased circulating resistin and heart failure, atherosclerotic CVD (all and hard) and coronary heart disease were noted even after adjustment for traditional cardiovascular risk factors.

- The association of resistin and cardiovascular events was greatest in the Hispanic population. 


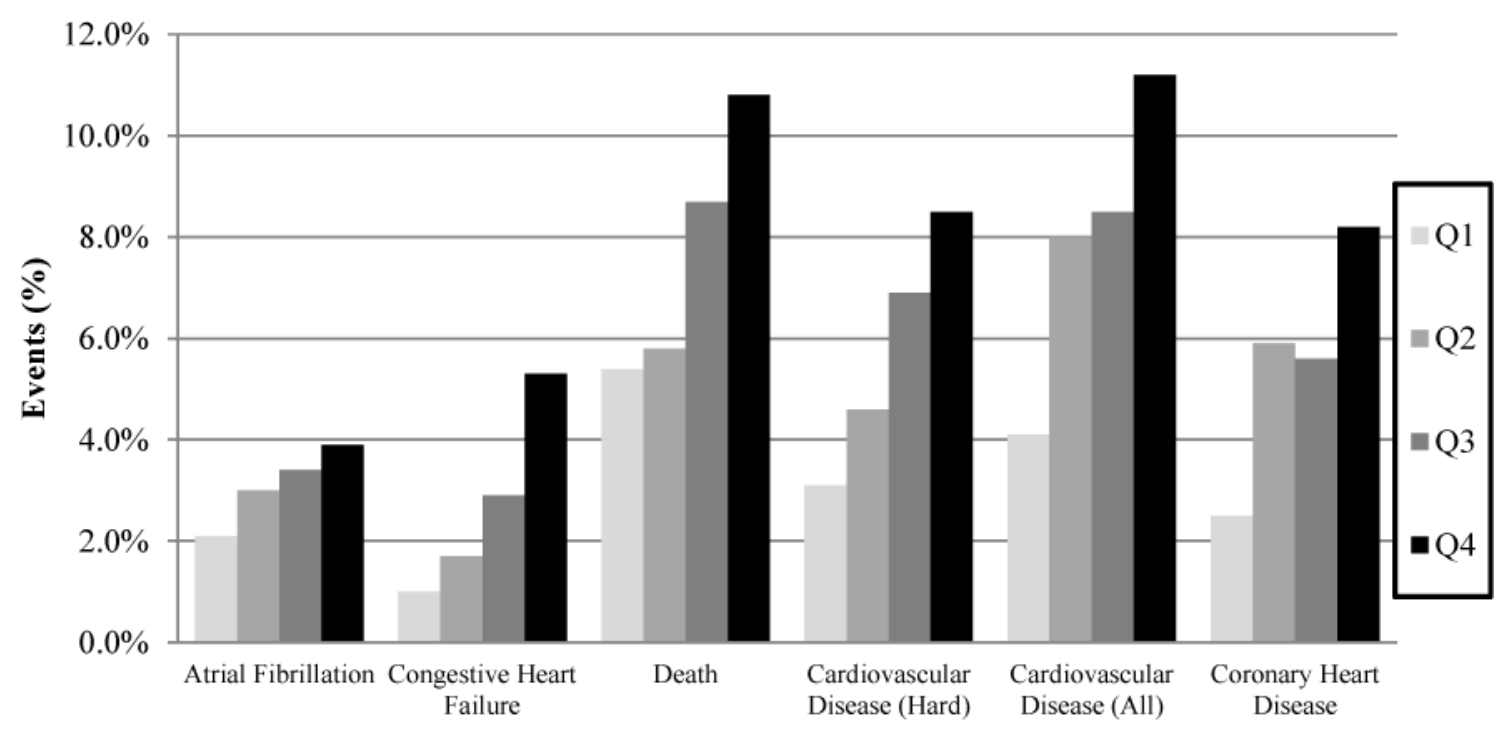

Figure 1. Total event numbers stratified by increasing quartiles of resistin

There is a graded increase in event rates across the first to the fourth quartile of resistin for $\mathrm{AF}, \mathrm{HF}$, death, hard CVD, CHD, and all CVD. 

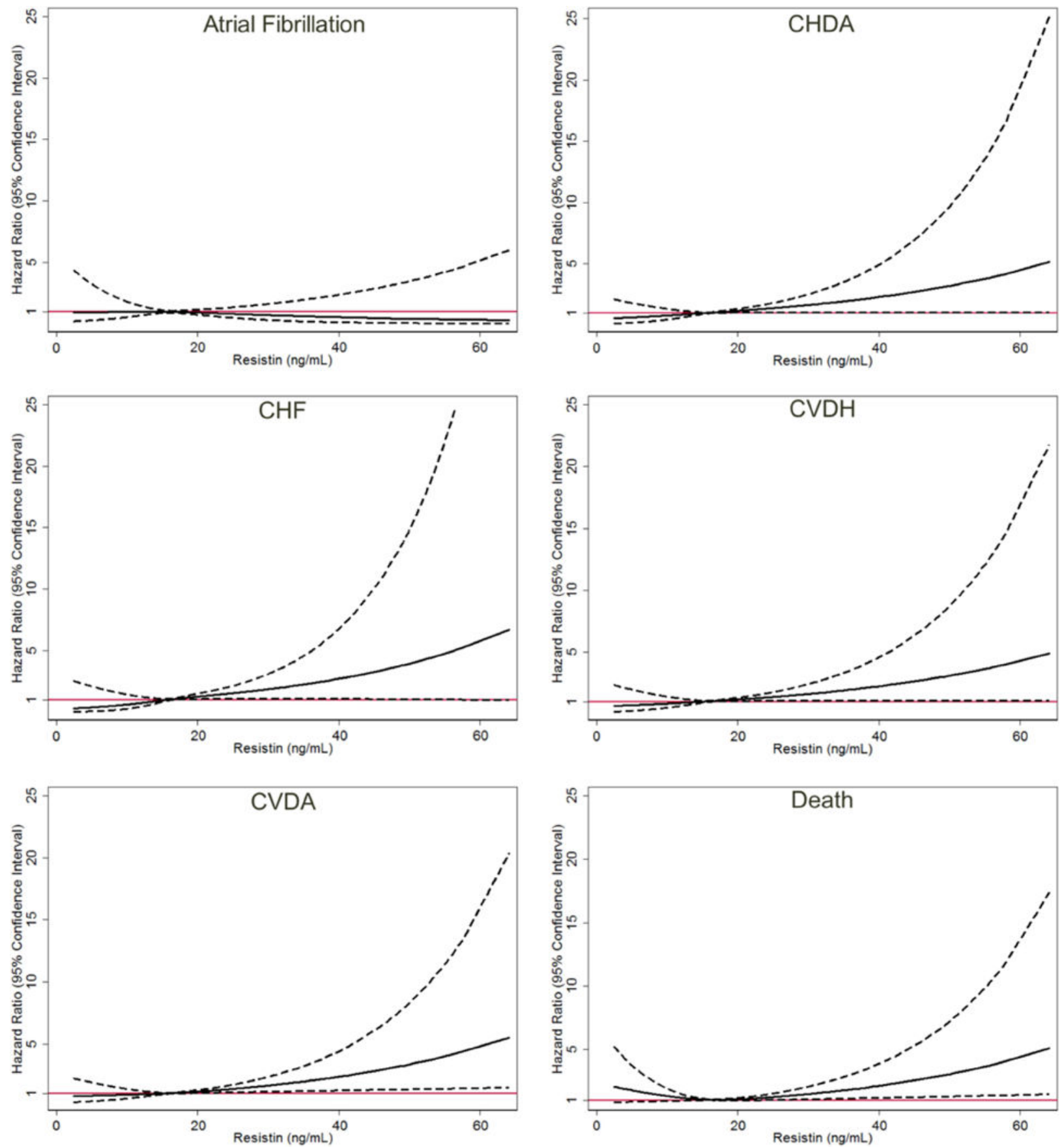

Figure 2.

Association of plasma resistin and outcomes using restricted cubic splines centered at the median value of resistin after adjustment for age, sex, hypertension, hypertension medications, diabetes mellitus, cigarette smoking, cholesterol, HDL-C, and lipid lowering therapy as in Model 2. A. Atrial fibrillation, B. CHD - all, C. HF, D. CVD - hard, E. CVD - all, F. death 
Table 1

Baseline Characteristics of Study Participants by Quartiles of Resistin

\begin{tabular}{|c|c|c|c|c|c|}
\hline & $\begin{array}{c}\text { Quartile 1, Range } \\
2.4-11.88(\mathrm{ng} / \mathrm{ml}) \\
\quad n=483\end{array}$ & $\begin{array}{c}\text { Quartile 2, Range } \\
\begin{array}{c}11.89-15.0(\mathrm{ng} / \mathrm{ml}) \\
n=475\end{array}\end{array}$ & $\begin{array}{c}\text { Quartile 3, Range } \\
15.1-19.13(\mathrm{ng} / \mathrm{ml}) \\
n=482\end{array}$ & $\begin{array}{c}\text { Quartile 4, Range } \\
\begin{array}{c}19.15-64.2(\mathrm{ng} / \mathrm{ml}) \\
n=473\end{array}\end{array}$ & $p$ Value \\
\hline Age (years) & $62.5 \pm 9$ & $63.7 \pm 9.4$ & $64.8 \pm 9.6$ & $67.2 \pm 9.9$ & $<0.01$ \\
\hline Female $(\%)$ & 50.9 & 45.7 & 51.5 & 53.1 & 0.12 \\
\hline Race: & & & & & $<0.01$ \\
\hline White (\%) & 31.7 & 47.0 & 45.2 & 36.6 & \\
\hline Chinese (\%) & 18.8 & 11.2 & 10.2 & 12.1 & \\
\hline Black (\%) & 24.0 & 16.0 & 16.4 & 27.5 & \\
\hline Hispanic (\%) & 25.5 & 25.9 & 28.2 & 23.9 & \\
\hline Income $>\$ 40 \mathrm{k}(\%)$ & 54.0 & 53.9 & 51.8 & 41.8 & $<0.01$ \\
\hline $\operatorname{BMI}\left(\mathrm{kg} / \mathrm{m}^{2}\right)$ & $27.3 \pm 4.9$ & $28.4 \pm 5.1$ & $28.1 \pm 5.2$ & $28.9 \pm 5.6$ & $<0.01$ \\
\hline Waist circumference $(\mathrm{cm})$ & $95.2 \pm 13.5$ & $99.0 \pm 13.6$ & $98.6 \pm 14.0$ & $100.3 \pm 15.1$ & $<0.01$ \\
\hline HOMA-IR (units) & $1.5 \pm 1.1$ & $1.7 \pm 1.4$ & $1.9 \pm 3.3$ & $2.1 \pm 2.2$ & $<0.01$ \\
\hline hs-CRP (mgl/L) & $1.2(0.6-2.5)$ & $1.5(0.7-2.9)$ & $1.5(0.7-3.3)$ & $1.9(0.9-4.7)$ & $<0.01$ \\
\hline Heart Rate & $64.7 \pm 9.5$ & $64.3 \pm 10.0$ & $64.8 \pm 9.8$ & $65.9 \pm 10.9$ & 0.08 \\
\hline Systolic blood pressure (mm Hg) & $122.4 \pm 20.4$ & $122.2 \pm 20.0$ & $123.4 \pm 21.3$ & $127.9 \pm 21.0$ & $<0.01$ \\
\hline Diastolic blood pressure (mm Hg) & $70.8 \pm 10.1$ & $69.8 \pm 9.9$ & $69.6 \pm 9.7$ & $70.3 \pm 9.8$ & 0.26 \\
\hline $\begin{array}{l}\text { Treatment with antihypertensive } \\
\text { medications }(\%)\end{array}$ & 36.3 & 43.0 & 39.1 & 52.8 & $<0.01$ \\
\hline Fasting Glucose (mmol/L) & $5.4 \pm 1.6$ & $5.4 \pm 1.6$ & $5.4 \pm 1.3$ & $5.4 \pm 1.5$ & 0.96 \\
\hline Diabetes $(\%) \mathrm{n}=265$ & 10.8 & 13.1 & 13.5 & 18.2 & $<0.01$ \\
\hline Hypoglycemic or insulin therapy $(\%)$ & 7.7 & 10.3 & 10.0 & 15.6 & $<0.01$ \\
\hline Cigarette smoking (\%): & $38.3,9.4$ & $44.4,11.6$ & $42.1,13.6$ & $44.5,10.6$ & 0.06 \\
\hline \multicolumn{6}{|l|}{ Former $\mathrm{n}=804$} \\
\hline \multicolumn{6}{|l|}{ Current $n=215$} \\
\hline Cholesterol levels (mmol/L) & $10.8 \pm 2$ & $10.6 \pm 1.9$ & $10.5 \pm 1.9$ & $10.4 \pm 1.9$ & 0.03 \\
\hline Triglycerides levels (mmol/L) & $5.9(4.2-8.2)$ & $6.4(4.3-9.6)$ & $6.5(4.3-9.3)$ & $6.5(4.6-9.7)$ & $<0.01$ \\
\hline HDL-C $(\mathrm{mmol} / \mathrm{L})$ & $3 \pm 0.9$ & $2.9 \pm 0.8$ & $2.8 \pm 0.8$ & $2.8 \pm 0.8$ & $<0.01$ \\
\hline LDL-C (mmol/L) & $6.4 \pm 1.8$ & $6.3 \pm 1.7$ & $6.2 \pm 1.7$ & $6.1 \pm 1.7$ & 0.07 \\
\hline Lipid Lowering Therapy (\%) & 24.7 & 25.7 & 21.6 & 25.5 & 0.42 \\
\hline Urinary Albumin: & 6.0 & 6.8 & 9.6 & 14.3 & $<0.01$ \\
\hline \multicolumn{6}{|l|}{ Microalbuminuria, $(\%) n=173$} \\
\hline Framingham risk score (\%) & $7.7 \pm 6.5$ & $8.2 \pm 6.7$ & $8.5 \pm 7.2$ & $9.8 \pm 7.6$ & $<0.01$ \\
\hline
\end{tabular}

Abbreviations: BMI, body mass index (calculated as weight in kilograms divided by the square of height in meters); HOMA-IR, homeostatic model assessment - insulin resistance; hsCRP, high sensitivity c-reactive protein; HDL-C, high density lipoprotein; LDL-C, low density lipoprotein 
Table 2

Hazard Ratios per Standard Deviation for Log-Transformed Resistin

\begin{tabular}{|c|c|c|c|c|c|c|}
\hline & \multirow[b]{2}{*}{ Events $(N, \%)$} & \multicolumn{5}{|c|}{ Hazard Ratio* per SD log [resistin] } \\
\hline & & Unadjusted & Model 1 & Model 2 & Model 3 & Model 4 \\
\hline$A F$ & $52,2.72 \%$ & $\begin{array}{c}1.1 \\
(0.9-1.5)\end{array}$ & $\begin{array}{c}1.0 \\
(0.7-1.3)\end{array}$ & $\begin{array}{c}0.9 \\
(0.7-1.2)\end{array}$ & $\begin{array}{c}0.9 \\
(0.7-1.3)\end{array}$ & $\begin{array}{c}0.9 \\
(0.7-1.2)\end{array}$ \\
\hline$H F$ & $58,3.12 \%$ & $\begin{array}{c}1.9 \\
(1.5-2.5)\end{array}$ & $\begin{array}{c}1.7 \\
(1.3-2.2)\end{array}$ & $\begin{array}{c}1.5 \\
(1.1-2.1)\end{array}$ & $\begin{array}{c}1.5 \\
(1.1-2.0)\end{array}$ & $\begin{array}{c}1.4 \\
(1.0-2.0)\end{array}$ \\
\hline CVD (hard) & $110,5.76 \%$ & $\begin{array}{c}1.5 \\
(1.3-1.8)\end{array}$ & $\begin{array}{c}1.4 \\
(1.1-1.7)\end{array}$ & $\begin{array}{c}1.3 \\
(1.1-1.6)\end{array}$ & $\stackrel{1.3}{(1.1-1.6)}$ & $\begin{array}{c}1.3 \\
(1.1-1.7)\end{array}$ \\
\hline$C V D($ all $)$ & $152,7.95 \%$ & $\begin{array}{c}1.5 \\
(1.2-1.7)\end{array}$ & $\begin{array}{c}1.4 \\
(1.2-1.6)\end{array}$ & $\begin{array}{c}1.3 \\
(1.1-1.5)\end{array}$ & $\begin{array}{c}1.3 \\
(1.1-1.5)\end{array}$ & $\begin{array}{c}1.3 \\
(1.1-1.6\end{array}$ \\
\hline$C H D$ & $106,5.55 \%$ & $\begin{array}{c}1.5 \\
(1.2-1.8)\end{array}$ & $\begin{array}{c}1.4 \\
(1.2-1.8)\end{array}$ & $\begin{array}{c}1.3 \\
(1.1-1.7)\end{array}$ & $\begin{array}{c}1.3 \\
(1.0-1.6)\end{array}$ & $\begin{array}{c}1.3 \\
(1.0-1.6)\end{array}$ \\
\hline Death & $147,7.69 \%$ & $\begin{array}{c}1.3 \\
(1.1-1.5)\end{array}$ & $\begin{array}{c}1.1 \\
(0.9-1.3)\end{array}$ & $\begin{array}{c}1.0 \\
(0.9-1.2)\end{array}$ & $\begin{array}{c}1.0 \\
(0.9-1.3)\end{array}$ & $\begin{array}{c}1.0 \\
(0.8-1.2)\end{array}$ \\
\hline
\end{tabular}

Model 1: Age, Sex, Race. Model 2: Model 1 + hypertension, hypertension medications, diabetes mellitus, cigarette smoking, total cholesterol, HDL cholesterol, lipid therapy. Model 3: Model 2 + BMI, HOMA-IR, log(hs-CRP). Model 4: Model 3 + leptin, adiponectin

Abbreviations: AF, Atrial Fibrillation; HF, Congestive Heart Failure; CVD, Cardiovascular Disease; CHD, Coronary Heart Disease

* Hazard Ratios are expressed per standard deviation of log-transformed resistin 
Table 3

Hazard Ratios per Standard Deviation for Log-Transformed Resistin, Stratified by Race

\begin{tabular}{|c|c|c|}
\hline & \multicolumn{2}{|c|}{ Hazard Ratio per SD log [resistin] } \\
\hline & Model 1 & Model 2 \\
\hline \multicolumn{3}{|l|}{ Caucasian } \\
\hline$A F$ & $0.8(0.5-1.2)$ & $0.7(0.4-1.1)$ \\
\hline$H F$ & $2.1(1.2-3.6)$ & $1.8(1.0-3.2)$ \\
\hline Death & $1.3(1.0-1.8)$ & $1.3(0.9-1.8)$ \\
\hline$C V D$ (hard) & $1.2(0.8-1.7)$ & $1.1(0.7-1.6)$ \\
\hline$C V D($ all $)$ & $1.2(0.9-1.6)$ & $1.1(0.8-1.5)$ \\
\hline$C H D$ & $1.4(1.0-2.0)$ & $1.3(0.9-1.9)$ \\
\hline \multicolumn{3}{|l|}{ Chinese } \\
\hline$A F$ & $0.8(0.3-2.0)$ & $0.3(0.0-2.6)$ \\
\hline$H F$ & $1.3(0.5-3.4)$ & $1.4(0.4-4.8)$ \\
\hline Death & $0.9(0.6-1.4)$ & $0.8(0.5-1.3)$ \\
\hline$C V D$ (hard) & $1.5(0.7-3.3)$ & $1.0(0.3-3.8)$ \\
\hline$C V D($ all $)$ & $1.6(0.8-3.1)$ & $1.4(0.6-2.9)$ \\
\hline$C H D$ & $1.6(0.8-3.1)$ & $1.4(0.6-2.9)$ \\
\hline \multicolumn{3}{|l|}{ Black } \\
\hline$A F$ & $1.0(0.7-1.6)$ & $1.0(0.6-1.5)$ \\
\hline$H F$ & $1.3(0.8-2.2)$ & $1.3(0.7-2.2)$ \\
\hline Death & $1.0(0.7-1.3)$ & $0.9(0.7-1.2)$ \\
\hline$C V D$ (hard) & $1.2(0.9-1.6)$ & $1.1(0.8-1.5)$ \\
\hline$C V D($ all $)$ & $1.1(0.8-1.6)$ & $1.1(0.7-1.5)$ \\
\hline$C H D$ & $2.9(0.8-1.7)$ & $1.1(0.7-1.7)$ \\
\hline \multicolumn{3}{|l|}{ Hispanic ${ }^{\dagger}$} \\
\hline$A F$ & $1.6(0.8-3.2)$ & $1.4(0.6-3.1)$ \\
\hline$H F$ & $2.2(1.4-3.7)$ & $1.9(1.1-3.4)$ \\
\hline Death & $1.1(0.7-1.6)$ & $0.9(0.6-1.4)$ \\
\hline$C V D$ (hard) & $2.3(1.6-3.3)$ & $2.1(1.4-3.1)$ \\
\hline$C V D($ all $)$ & $2.0(1.4-2.7)$ & $1.8(1.2-2.5)$ \\
\hline CHD & $1.8(1.2-2.8)$ & $1.5(1.0-2.4)$ \\
\hline
\end{tabular}

Model 1: Age, Sex. Model 2: Model $1+$ hypertension, hypertension medications, diabetes mellitus, cigarette smoking, total cholesterol, HDL cholesterol, lipid therapy.

\section{Interaction Terms:}

* Overall race interaction term is significant for CVD (hard) after adjustments for variables in model 1 ( $\mathrm{p}=0.02) \&$ model 2 ( $\mathrm{p}=0.02$ ). This appears to be driven by more significant findings in Hispanics.

${ }^{\dagger}$ Interaction term for Hispanic vs. non-Hispanic X SD $\log$ [resistin] is significant for both CVD (hard) and CVD (all) after adjustment for variables in model $1(\mathrm{p}=0.01) \&$ model $2(\mathrm{p}=0.03)$.

Abbreviations: AF, Atrial Fibrillation; HF, Congestive Heart Failure; CVD, Cardiovascular Disease; CHD, Coronary Heart Disease. 